\title{
Phonological but not auditory discrimination is impaired in dyslexia
}

\author{
Isabella Paul, Christof Bott, Sabine Heim, Christian Wienbruch and Thomas R. Elbert \\ University of Konstanz, Clinical Psychology, Konstanz, Germany
}

Keywords: dyslexia, MEG, MMF, phonological

\begin{abstract}
Deficient phonological skills are considered to be a core problem in developmental dyslexia. Children with dyslexia often demonstrate poorer performance than non-impaired readers when categorizing speech-sounds. Using the automatic mismatch response, we show that in contrast to this deficit at the behavioural level, neurophysiological responding in dyslexic children indicates their ability to automatically discriminate syllables. Therefore, the phonological deficit is unlikely to be caused by a temporal deficit or by a noisy functional organization in the respective representational cortex. We obtained measures of reading, spelling and categorical speechperception from 58 dyslexic children and 21 control children. The children also participated in magnetoencephalographic measurements while being stimulated acoustically with the syllables $/ \mathrm{ba} /$ and $/ \mathrm{da} /$ in an oddball paradigm. Mismatch field (MMF) amplitudes between standard and deviant stimuli were obtained. Dyslexic children performed more poorly than control children on all test measures. However, the groups did not differ in MMF amplitude or latency. No correlations were found between MMF amplitudes and behavioural performance.These results were obtained with a large sample size and thus speak robustly against a general deficit in auditory discrimination in dyslexia. These results are compatible with the idea that decoding difficulties occur later in the processing stream where access to the phonological lexicon is attempted.
\end{abstract}

\section{Introduction}

Reduced phonological awareness (the reduced ability to discriminate speech-sounds in spoken words) is considered to be a core-deficit in dyslexia (Vellutino, 1987; Elbro et al., 1996; Swan \& Goswami, 1997; Ramus et al., 2003). Phonological skills are not only assumed to be a prerequisite for speech-perception, but also for learning the correspondence between graphemes and phonemes, which is crucial for reading and writing (Stanovich, 1988; Rack et al., 1992; Torgesen et al., 1994; Shaywitz, 1996; Ramus et al., 2003). Children with dyslexia perform more poorly than control children in categorizing speech-sounds (Mody et al., 1997; Adlard \& Hazan, 1998; Serniclaes et al., 2001). Most dyslexic children have problems distinguishing consonant-vowel (CV) syllables (Manis et al., 1997). This result seems to be related to their deficiency in processing stop-consonants (Manis et al., 1997; McAnally et al., 1997). Stop-consonants are characterized by 'fast frequency changes' within a timeframe of milliseconds. Therefore, some authors have argued that the processing deficiencies in dyslexia might not be purely phonological, but a result of an underlying temporal (auditory) processing deficit (Tallal, 1980; Farmer \& Klein, 1995; Tallal et al., 1996; Wright et al., 1997; Temple et al., 2001).

Mismatch negativity (MMN) and its magnetic counterpart mismatch field (MMF) recorded during presentation of language-specific speech-sound stimuli may help to clarify this question. Kraus and colleagues (Kraus et al., 1996) investigated the MMN in 21 control children with good abilities to discriminate a /da/-/ga/ contrast, as well as 21 children with poor abilities [including children with

Correspondence: Dr I. Paul, as above.

E-mail: Isabella.Paul@uni-konstanz.de

Received 19 September 2006, revised 24 August 2006, accepted 30 August 2006 learning disabilities and attention deficit hyperactivity disorder (ADHD)]. Participants were selected from a pool of 91 children with learning disabilities and 90 control children. The stimuli that were used in an oddball paradigm were selected to be difficult to discriminate for listeners with normal abilities. A significantly larger MMN, with a longer duration, was found in children who could discriminate $/ \mathrm{da} /-/ \mathrm{ga} /$ accurately than in children who were inaccurate. Further, a correlation was found between discrimination performance and both MMN area and duration. The authors concluded that the behavioural discrimination performance of children with learning disabilities is mirrored by an electrophysiological measure that originates in the auditory pathway. The same stimuli as the ones used by Kraus and colleagues were also presented to 74 children with learning disabilities and 46 control children by Banai et al. (2005). Additionally, the authors investigated brainstem timing. They only found a significant MMN in the control children, and $40 \%$ of the children with learning disabilities were also characterized by abnormal brainstem timing. These results suggest that in some children with learning disabilities phonological and other difficulties might be related to even more basic processing deficiencies in the auditory pathway.

MMN after auditory stimulation has also been investigated in purely dyslexic populations. Schulte-Körne and his co-workers (Schulte-Körne et al., 1998) found an attenuated MMN for a dyslexic sample compared with controls after stimulation with CV syllables (standard $/ \mathrm{da} /$, deviant $/ \mathrm{ba} /$ ), but not after stimulation with pure tones (standard $1000 \mathrm{~Hz}$, deviant $1050 \mathrm{~Hz}$ ). This result was interpreted as evidence that dyslexia is a speech-related phenomenon that does not extend to other domains of auditory processing. In the study by Schulte-Körne et al. (1999), a sequence of four tones differing in frequency and duration was presented as standard stimulus. Deviants 
only differed in the duration of the second and last tone (which had the same frequency). In this paradigm, the dyslexic subjects did show an attenuated MMN also for non-speech-sounds. Because the only difference between standard and deviant stimuli lay in the temporal structure of the tone pattern, the authors concluded that temporal auditory processing might be deficient in dyslexia. These results suggest that dyslexia is related to processing difficulties of speechsounds as well as temporally complex non-speech-sounds. This implies that the processing deficit in dyslexia is not only phonological in nature.

Kujala and colleagues (Kujala et al., 2003) also found support for an auditory temporal discrimination deficit in dyslexia. Adult dyslexic subjects and control subjects were presented with tone patterns in an oddball paradigm. In the deviant condition, the standard tone-pair could either be preceded or followed by a third tone. The groups did not differ in MMN amplitude when the deviant stimulus was preceded by a third tone. MMN amplitude was reduced in the dyslexic subjects, however, when the deviant stimulus was followed by a third tone. The authors interpreted this finding as a backward masking interference with sound perception in dyslexia and thus as support for the notion that dyslexic subjects have auditory discrimination problems at a basic level.

In conclusion, the current literature suggests that reduced MMN amplitudes in dyslexia are a robust feature. They have been shown in experiments using speech stimuli indicating that dyslexia is characterized by a low-level deficit in auditory speech-perception. Reduced MMNs have also been demonstrated in experiments that presented non-speech stimuli that were either temporally complex or created the potential for backward masking. This was interpreted as evidence for a perceptual deficit that might involve even more basic levels of auditory analysis.

Based on these findings, the present study aimed: (a) to investigate the relationship between MMF (as a measure of basic auditory speech processing) and several behavioural tasks ranging from reading and spelling to phonological abilities and categorical speech-perception; and (b) to investigate the differential influence of three dyslexia training programmes that focused on different levels of processing. We measured behavioural performance in various tests as well as the MMF in a comparatively large sample of dyslexic and control children before and after training. Surprisingly, we did not find any group differences in MMF amplitude. This was particularly unexpected as the dyslexic children performed significantly worse than the control group in a test of the ability to identify the syllables $/ \mathrm{ba} /$ and $/ \mathrm{da} /$. In the present paper, only results obtained before training will be presented. Post-training data will be presented elsewhere.

\section{Materials and methods}

\section{Generation of the sample, test measures}

The participating children were contacted through 14 primary schools in or around Konstanz, Germany, and attended either 3rd or 4th grade. Schools were asked to name children with considerable problems in reading and spelling, as well as children without any such difficulties. In order to classify the children objectively as dyslexic, all participants underwent a test-battery that was designed to assess a variety of abilities ranging from spelling and reading to phonological abilities (Table 1). If a child, who was suggested to be dyslexic by the teacher, was not worse than the normative sample in the standardized spelling test $(<50 \mathrm{~T}$-values), he/she was excluded from the study $(n=2)$. Control children who performed significantly worse than the normative sample in the spelling test were also excluded $(n=5)$. It was required that there was a discrepancy between the dyslexic children's IQ and performance in the spelling test of at least 1.5 standard deviations.

\section{Subjects}

Fifty-eight children with dyslexia and 21 children without any reading or spelling deficits participated in the study after giving informed consent. The groups were similar in age (controls $9.2 \pm 0.2$ years, dyslexics $9.1 \pm 0.1$ years), handedness (controls 95\%, dyslexics $84 \%$ right-handed) and gender (controls 67\%, dyslexics 71\% male). Ethical approval of the study was given through the Ethical Review Board of the Konstanz University.

\section{Study design}

Each child underwent the test-battery (see Table 1) that was split into three 45-min sessions on separate days. Following the behavioural tests, the magnetoencephalogram (MEG) was recorded for each child.

\section{Stimulation in the MEG}

The two CV speech stimuli were the 5th and 8th members of a 10-item continuum of synthetic syllables that ranged from $/ \mathrm{ba} /$ to $/ \mathrm{da} /$. Both stimuli were located close to the phoneme boundary, with item 5 generally heard as /ba/ and item 8 generally heard as /da/. The syllables had been synthesized to resemble a male voice. They had a duration of $250 \mathrm{~ms}$ and differed only in the onset frequencies of the second (F2) and third (F3) formant transitions. Starting frequencies of $\mathrm{F} 2$ and F3 were 1365 and $2337 \mathrm{~Hz}$ for /ba/, and 1567 and $2515 \mathrm{~Hz}$ for $/ \mathrm{da} /$. The formant transitions lasted $40 \mathrm{~ms}$ and reached steadystate frequencies of 1340 and $2400 \mathrm{~Hz}$, respectively.

In an auditory oddball task, the syllable /ba/ was used as the standard stimulus and $/ \mathrm{da} /$ as the deviant. In total, 500 stimuli were presented with a constant interstimulus interval (ISI) of $500 \mathrm{~ms}$. Occurrence rates were $85 \%$ and $15 \%$ for $/ \mathrm{ba} /$ and $/ \mathrm{da} /$, respectively. Stimuli were presented pseudo-randomly. Data epochs with standard stimuli occurring directly after a deviant were not analysed.

\section{MEG recordings}

Recording was done with a 148-channel magnetometer (MAGNES ${ }^{\mathrm{TM}}$ $2500 \mathrm{WH}, 4 \mathrm{D}$ Neuroimaging, San Diego, USA). Subjects were lying supine in a comfortable position in the magnetically shielded room (Vakuumschmelze Hanau). Auditory stimuli were presented through ear tubes at a level that was $60 \mathrm{~dB}$ above each subject's threshold for detecting the stimuli. Frequency characteristics of the tube phones were linear up to $6000 \mathrm{~Hz}$. For artefact control, eye movements (EOG) were recorded from four electrodes attached to the left and right outer canthus and above and below the right eye, as well as cardiac activity (electrocardiogram, ECG) via two electrodes, one on each forearm. A SynAmps amplifier (Neuroscan) served for the recording of EOG and ECG. A video camera installed inside the chamber allowed monitoring of the subject's behaviour and compliance throughout the experiment.

Subjects were instructed to not pay any attention to the syllables they would hear. To distract attention, a silent video was screened onto a white projection field at the ceiling of the chamber using a video projector (JVC ${ }^{\mathrm{TM}}$, DLA-G11E) and a mirror system. Data were recorded with a real high-pass filter of $0.1 \mathrm{~Hz}$ and a sampling rate of $508.63 \mathrm{~Hz}$ (bandwidth $100 \mathrm{~Hz}$ ). Recording was continuous. 
TABLE 1. Behavioural test-battery assessing non-verbal intelligence and literacy skills

\begin{tabular}{|c|c|c|}
\hline Skills and tests used & References & Notes \\
\hline \multicolumn{3}{|l|}{ Non-verbal intelligence } \\
\hline $\begin{array}{l}\text { Standard Progressive Matrices } \\
\text { (SPM) }\end{array}$ & (Heller et al., 1998) & \\
\hline \multicolumn{3}{|l|}{ Spelling } \\
\hline $\begin{array}{l}\text { Diagnostischer Rechtschreibtest } \\
\text { (DRT for grades } 3 \text { and } 4 \text { ) }\end{array}$ & (Grund et al., 1994; Müller, 1997): & Requires children to complete gap sentences by filling in spoken words \\
\hline \multicolumn{3}{|l|}{ Reading } \\
\hline Zürcher Lesetest, ZLT & (Grissemann, 2000) & Requires participants to read aloud lists of words and small passages \\
\hline Word-reading test & (This study) & Contains 50 words varying in complexity to be read aloud \\
\hline \multicolumn{3}{|l|}{ Phonological abilities } \\
\hline Pseudoword-reading test & (This study) & Comprises 50 pseudowords varying in complexity to be read aloud \\
\hline Mottier test & (Welte, 1981) & Assesses pseudoword-repetition performance \\
\hline Phonetic dictation & (Findeisen \& Melenk, 1991) & $\begin{array}{l}\text { Requires children to write a story encompassing phonetically regular } \\
\text { words from dictation }\end{array}$ \\
\hline \multirow[t]{3}{*}{ Categorical perception } & (This study) & $\begin{array}{l}\text { The listener is asked to identify stimuli of a } 10 \text {-item } / \mathrm{ba} /-/ \mathrm{da} / \\
\text { continuum into the phonemic categories. Syllables differ only in the } \\
\text { onset frequencies of the second and third formants ranging in linear } \\
\text { steps from the } / \mathrm{ba} / \text { endpoint (item } 1 \text { ) to the } / \mathrm{da} / \text { endpoint (item } 10 \text { ). } \\
\text { Categorical perception performance is quantified by using the following } \\
\text { formula, with } a \text { representing the number of responses for } / \mathrm{ba} / \text { and } b \\
\text { the number of responses for } / \mathrm{da} / \text { : }\end{array}$ \\
\hline & & $f=\sqrt{ } \sum_{i=1}^{10}\left(a_{i}-b_{i}\right)^{2}$ \\
\hline & & $\begin{array}{l}\text { A high perception index indicates reliable and correct categorization } \\
\text { of the syllables. }\end{array}$ \\
\hline
\end{tabular}

\section{Data analysis}

Data were noise-reduced and corrected for cardiac activity (4D Neuroimaging). For each subject, data epochs with a 200-ms baseline and a post-trigger window of $800 \mathrm{~ms}$ were generated. Epochs containing artefacts (signals $>120 \mu \mathrm{V}$ in the EOG and signals $>5 \mathrm{pT}$ in the MEG-channels) were rejected. The remaining epochs were averaged separately for the standard condition and the deviant condition. The resulting average files were $20-\mathrm{Hz}$ low-pass-filtered and baselinecorrected. Figure 1 shows the channel groups used for analysis:
The following formula was used for calculating the root mean square (RMS) amplitude for each time point in different channel groups:

$$
\sqrt{\text { channels }} \frac{\sum_{\text {Amplitude value })^{2}}}{\text { Number of channels }}
$$

Standard left- and right-channel groups (Fig. 1) were chosen, as they are considered to cover most activity generated in temporal regions. The left fronto-temporal group was chosen, because it showed the

\section{left and right temporal}

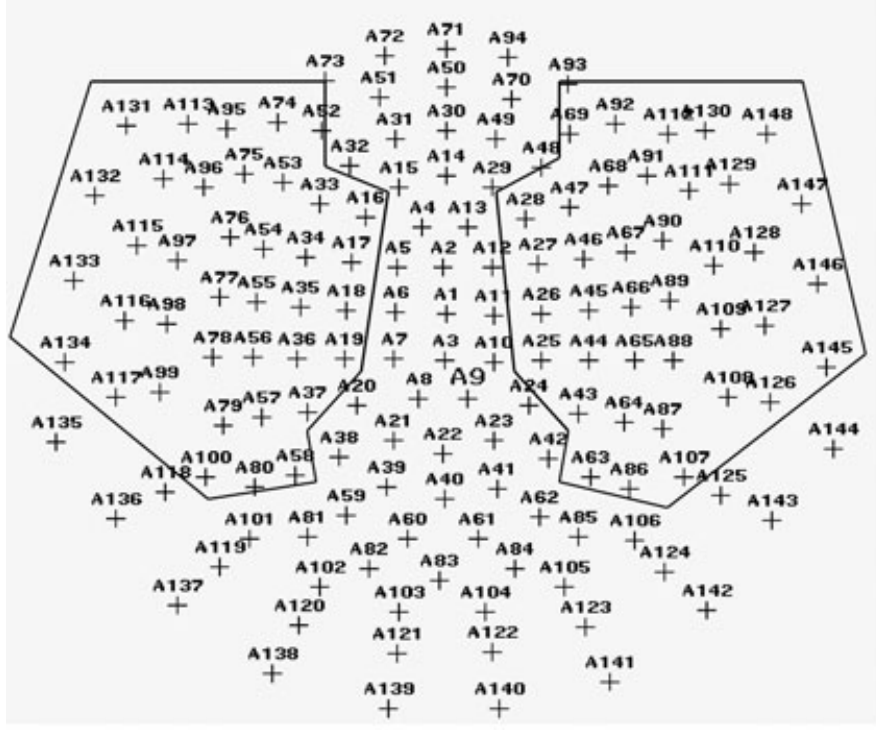

\section{left fronto-temporal}

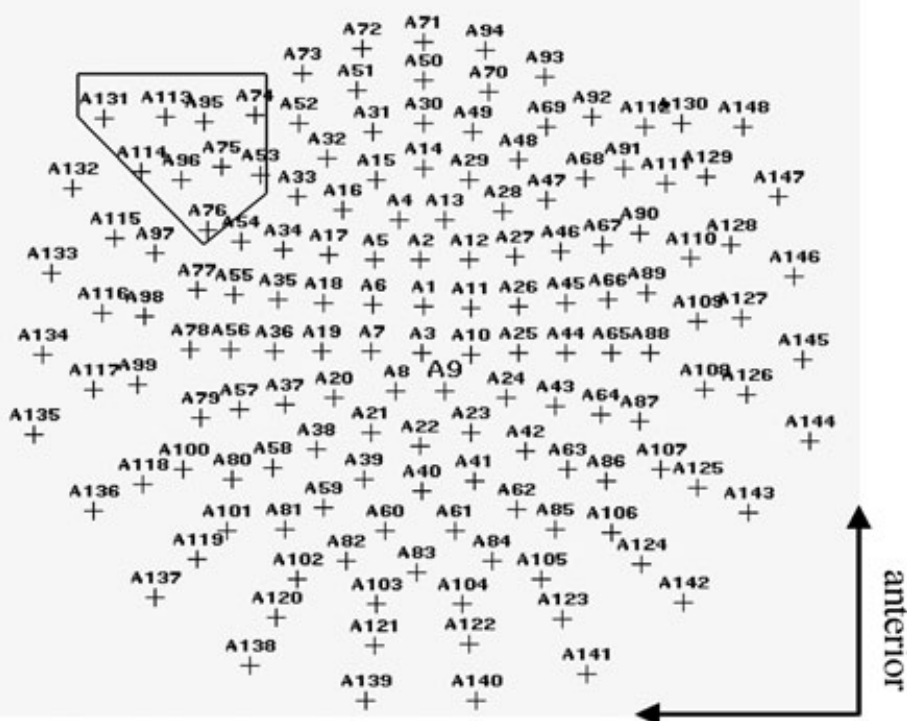

left

FIG. 1. Channel groups used for data analysis. 
TABLE 2. Performance in the behavioural tests

\begin{tabular}{|c|c|c|c|c|}
\hline & Control & Dyslexic & $F_{1,77 \text {-value }}$ & $P$-value \\
\hline SPM $(T)$ & $61.8 \pm 13.73$ & $51.4 \pm 10.86$ & 12.17 & 0.0008 \\
\hline $\operatorname{DRT}(T)$ & $57.8 \pm 5.97$ & $36.8 \pm 5.86$ & 195.49 & $<0.0001$ \\
\hline ZLT correctness (\%correct) & $96 \pm 2$ & $87 \pm 9$ & 22.82 & $<0.0001$ \\
\hline ZLT reading time (s/no. of words) & $0.68 \pm 0.17$ & $1.43 \pm 0.70$ & 23.65 & $<0.0001$ \\
\hline Word reading correctness (\%correct) & $90 \pm 7$ & $74 \pm 12$ & 32.81 & $<0.0001$ \\
\hline Word reading time $(\mathrm{s})$ & $71.2 \pm 18.51$ & $169.4 \pm 78.39$ & 30.95 & $<0.0001$ \\
\hline Pseudoword reading correctness (\%correct) & $76 \pm 13$ & $52 \pm 17$ & 37.14 & $<0.0001$ \\
\hline Pseudoword reading time (s) & $116.7 \pm 26.31$ & $217.7 \pm 122.63$ & 13.91 & 0.0004 \\
\hline Mottier test (\%correct) & $84 \pm 9$ & $66 \pm 17$ & 22.66 & $<0.0001$ \\
\hline Dictation (\%correct) & $92 \pm 5$ & $75 \pm 13$ & 37.09 & $<0.0001$ \\
\hline Categorical perception index $\left(F_{1,73}\right)$ & $30.7 \pm 2.83$ & $24.7 \pm 6.25$ & 17.13 & $<0.0001$ \\
\hline
\end{tabular}

Means $\pm \mathrm{SD}$ are displayed along with $F$ - and $P$-values of one-way-ANOvA. Dyslexic children performed more poorly than control children on all test measures.

clearest amplitude differences between standards and deviants. Mean amplitudes for the standard and deviant condition were calculated by averaging the RMS-values in three different time-windows $[\mathrm{W} 1=180-230 \mathrm{~ms} ; \mathrm{W} 2=230-280 \mathrm{~ms} ; \mathrm{W} 3=280-330 \mathrm{~ms}$ (see Fig. 3, results section)]. The mean amplitudes of the standard condition were subtracted from the mean amplitudes of the deviant condition yielding a measure of amplitude difference (MMF) between deviant and standard condition.

\section{Statistical analysis}

Difference values were analysed statistically via mixed models (using the PROC MIXED module of SAS ${ }^{\mathrm{TM}}$ ) for the individual channel groups (left temporal, left fronto-temporal, right temporal). MMF was used as the dependent variable. GROUP (Control, Dyslexic) and WINDOW (W1, W2, W3) were fixed effects; PAT (subject) nested within GROUP was used as a random factor.

\section{Behavioural data}

Behavioural performance in measures of non-verbal intelligence and literacy skills was analysed by computing one-way ANOVAs with GROUP (Control, Dyslexic) as a categorical predictor. Table 2 shows the mean test scores and $F$ statistics.

Further, correlations were calculated between MMF (averaged across the time-windows) and the scores in the behavioural tests.

\section{Results}

\section{Behavioural data}

A main effect of GROUP was found in all tests. The performance of the control children was better than the performance of the dyslexic children on measures of non-verbal intelligence and literacy skills (Table 2). Importantly, dyslexic children performed significantly worse than control children in categorizing the syllables $/ \mathrm{ba} /$ and $/ \mathrm{da} /$ $\left(F_{1,73}=17.13, P<0.0001\right)$.

\section{MMF data}

Figure 2A displays the grand mean butterfly plot of all MEG channels in the standard condition for the control children. As can be seen in Fig. 2B, an earlier, mainly left hemispheric bipolar field is followed by a later bilateral field pattern with reversed polarity. The earlier component is orientated upwards (representing a positive deflection in electroencephalogram, EEG), the later component (starting $~ 180 \mathrm{~ms}$ ) is orientated downwards (representing a negative deflection in EEG).

Figure 3 shows the RMS-waveforms of the standard and deviant condition for all control and dyslexic children at the left frontotemporal channel group between 80 and $330 \mathrm{~ms}$.

Statistical analysis was performed for the component starting at $180 \mathrm{~ms}$, because it was clearly identifiable in most of the cases (see Fig. 3). Analysis was done in 50-ms time-windows, the first window covering the rising slope of the component (180-230 ms), the second window covering the peak (230-280 ms), the third window covering the falling slope (280-330 ms). The results for the three different channel groups (left and right temporal, left fronto-temporal) were as follows.

Neither the main effects of GROUP or WINDOW, nor the interaction GROUP $\times$ WINDOW were significant in the mixed-model analysis for either channel group (Table 3).

Thus, data from the three time-windows were averaged, and a twoway analysis of variance (ANOVA) was computed with MMF as the dependent variable, GROUP (Control, Dyslexic) as a categorical predictor and CHANNELGROUP (left, left fronto-temporal, right) as a repeated measure. Figure 4 shows scatter plots of the mean data across time-windows.

MMF amplitudes did not differ significantly between control and dyslexic children in either channel group $\left(F_{1,77}=0.80, P=0.38 \mathrm{left}\right.$ temporal; $F_{1,77}=0.15, P=0.70$ left fronto-temporal; $F_{1,77}=0.38$, $P=0.54$ right temporal).

Some children in both groups did not have positive MMF values, meaning that the deviant waveforms were not higher in amplitude than

TABLE 3. Results of mixed models analysis

\begin{tabular}{lll}
\hline $\begin{array}{l}\text { Channel group } \\
\text { and effect }\end{array}$ & $F$-value & $P$-value \\
\hline Left temporal & & \\
$\quad$ GROUP $(1,77)$ & 0.8 & 0.38 \\
WINDOW (2154) & 0.56 & 0.57 \\
GROUP $\times$ WINDOW $(2,154)$ & 0.66 & 0.52 \\
Left fronto-temporal & & \\
GROUP $(1,77)$ & 0.15 & 0.7 \\
WINDOW (2154) & 0.44 & 0.65 \\
GROUP $\times$ WINDOW $(2,154)$ & 0.86 & 0.42 \\
Right temporal & & \\
GROUP $(1,77)$ & 0.38 & 0.54 \\
WINDOW (2154) & 0.11 & 0.9 \\
GROUP $\times$ WINDOW $(2,154)$ & 0.27 & 0.76 \\
\hline
\end{tabular}



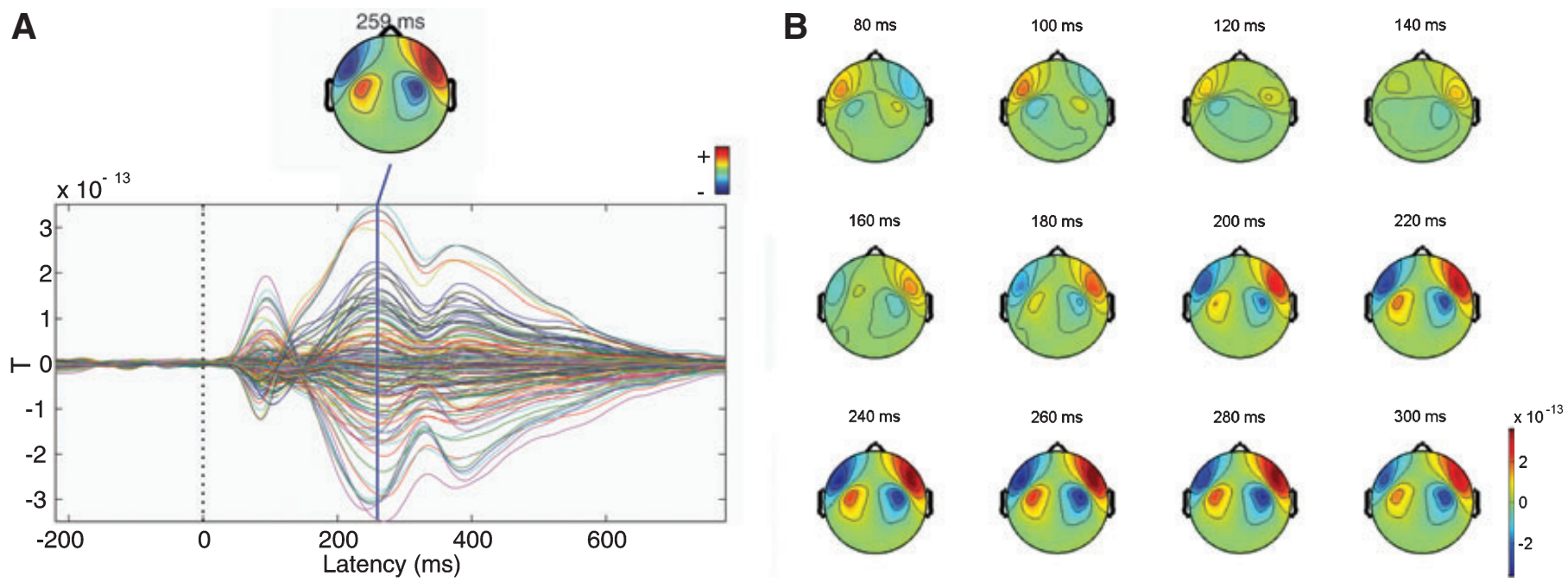

FIG. 2. (A) Grand mean of the standard condition (control children). The butterfly plot shows all MEG channels. (B) Topography maps of the grand mean in the standard condition.
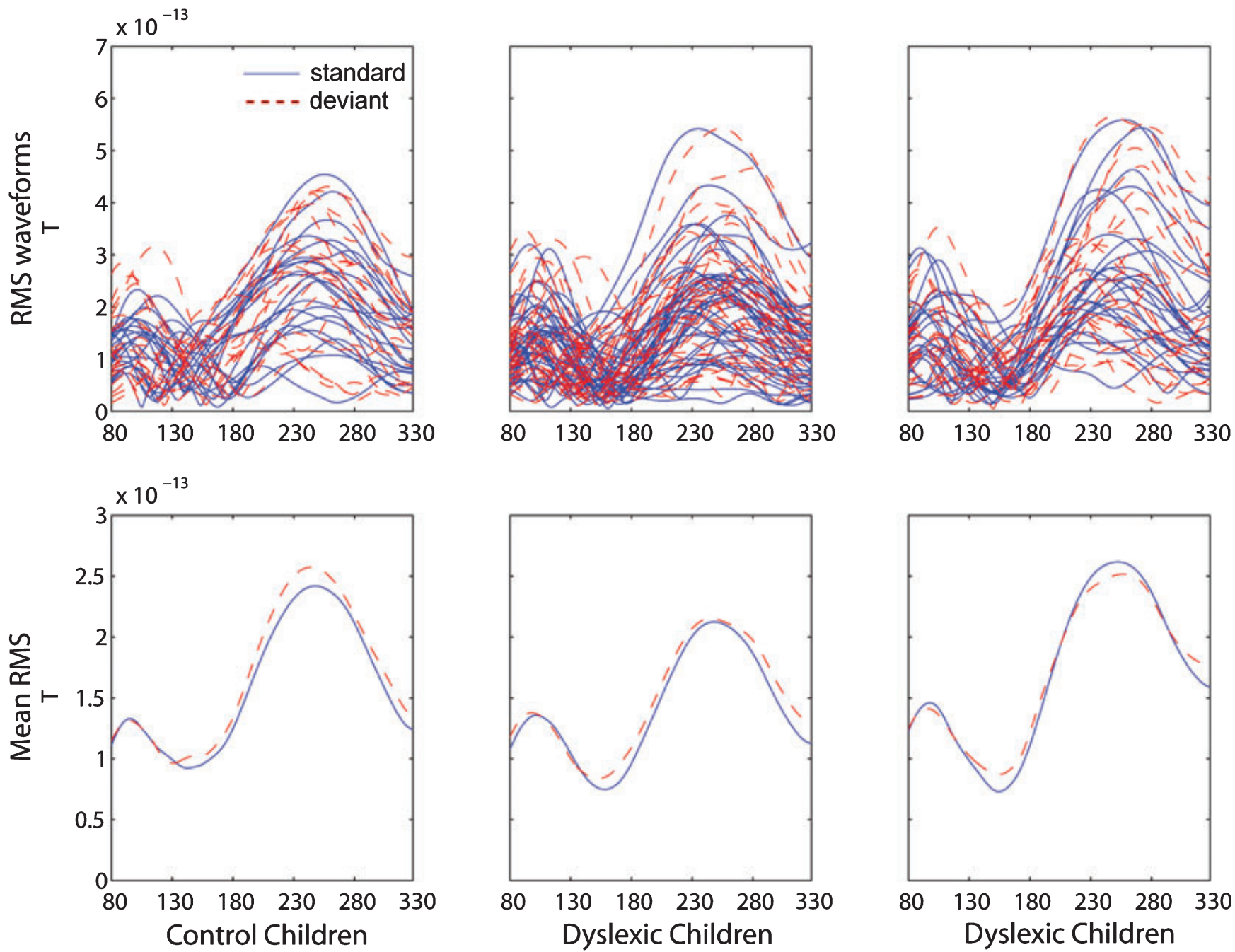

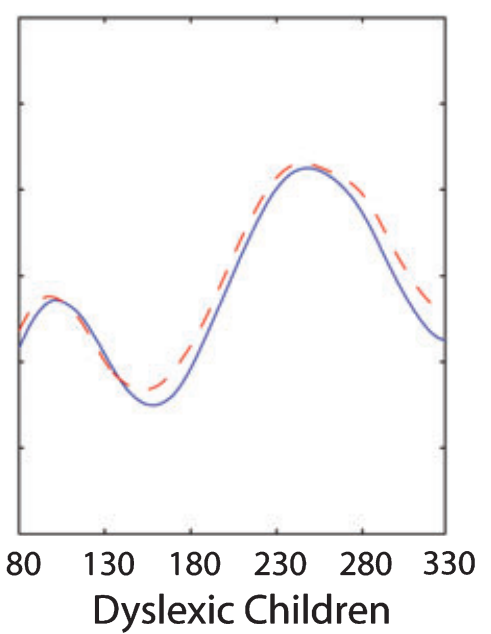

Good Categorical Perception

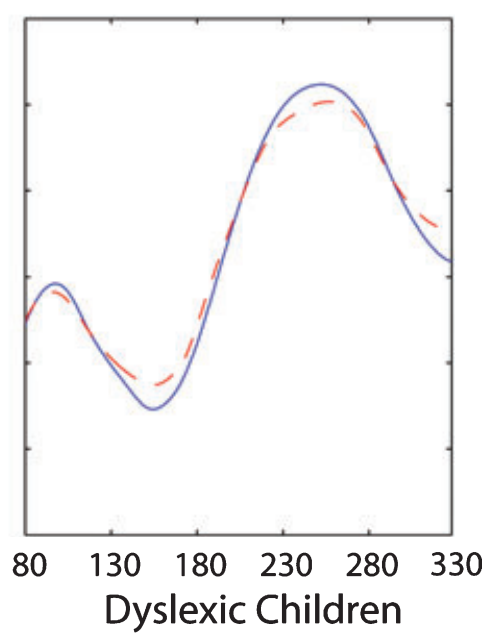

Poor Categorical Perception

FIG. 3. Root mean square (RMS) course of standards and deviants for control and dyslexic children. Bottom graphs display group means. 
Left Temporal Channels

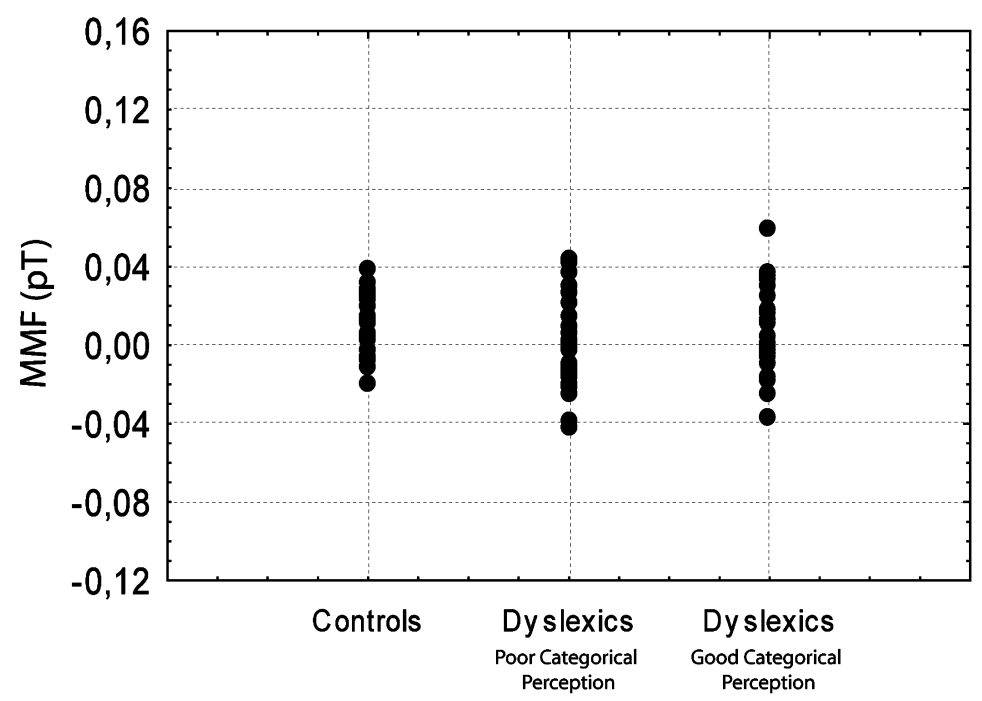

Right Temporal Channels

\section{Left Fronto-Temporal Channels}

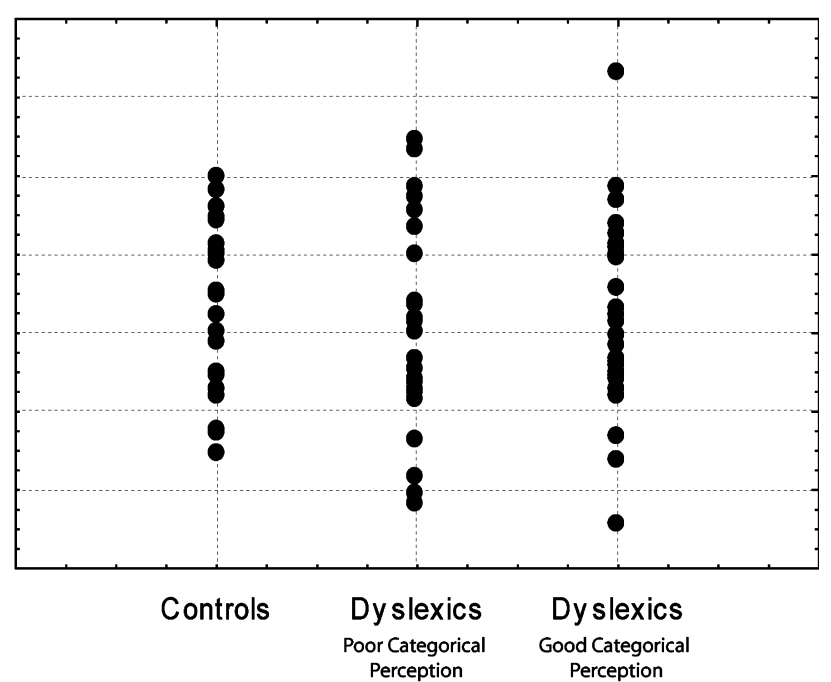

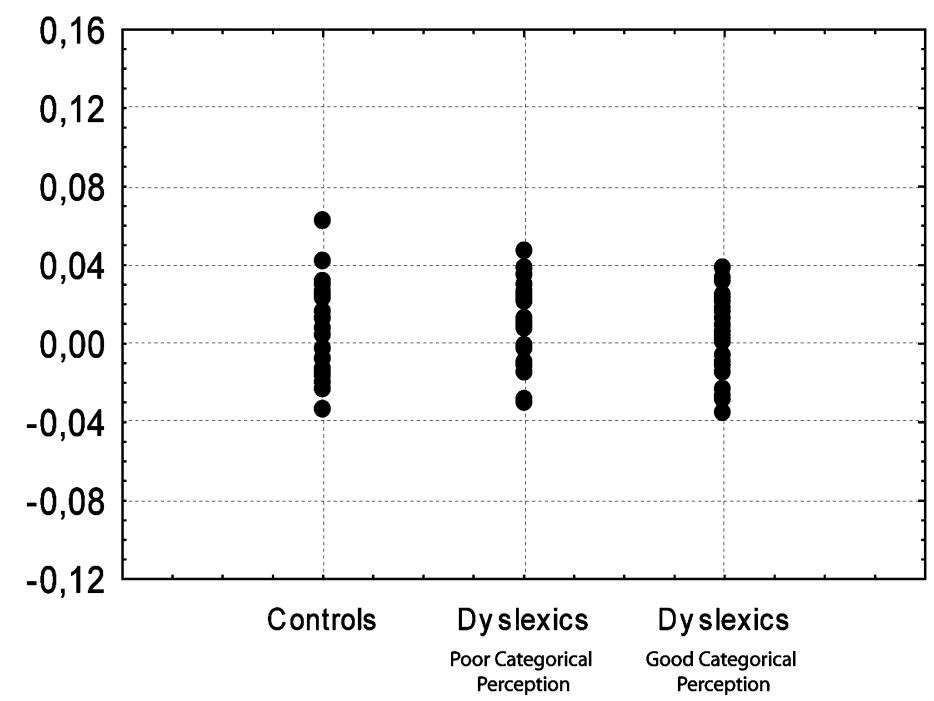

FIG. 4. Scatter plots of mismatch field (MMF) values (deviant-standard) in the three channel groups. Data are subdivided into control children, good dyslexic categorical perceivers and poor dyslexic categorical perceivers.

the standard waveforms. Thus, we performed another analysis only with those children who had positive MMF values (15 controls, 31 dyslexics left temporal; 12 controls, 32 dyslexics left fronto-temporal; 13 controls, 37 dyslexics right temporal). The groups did not differ in any channel group $\left(F_{1,44}=0.90, P=0.35\right.$ left temporal; $F_{1,42}=0.01, P=0.91$ left fronto-temporal; $F_{1,48}=2.20, P=0.14$ right temporal).

We performed a further analysis assuming that negative MMF values also represent the detection of deviant sounds - in a reversed fashion. Thus, we used absolute values of negative MMF amplitudes for statistical analysis. Again, the groups did not differ in any channel group $\left(F_{1,77}=0.60, P=0.44\right.$ left temporal; $F_{1,77}=0.06, P=0.81$ left fronto-temporal; $F_{1,77}=1.60, P=0.21$ right temporal).

Finally, we divided the dyslexic children based on their performance in categorical perception. The children who scored lower than 25 (lowest score in the control group) were classified 'poor' $(n=25)$, the children who performed better than 25 were classified 'good' $(n=29$, Fig. 5). We calculated another ANOVA, where we only compared control children with poor categorical perceivers. Again, the groups did not differ in any channel group $\left(F_{1,44}=1.997, P=0.16 \mathrm{left}\right.$ temporal; $F_{1,44}=0.33, P=0.57$ left fronto-temporal; $F_{1,44}=0.13$, $P=0.72$ right temporal).

In order to test if the stimuli that were used in the MEG experiment (the 5th and 8th items from the continuum of synthetic syllables) were too easy to discriminate, we compared the children's categorical perception performance (number of responses to $/ \mathrm{ba} /$ and $/ \mathrm{da} /$, respectively) at these steps. At step 5 , control children performed $89 \%$ correct while good categorical perceivers performed $79 \%$ correct, and poor categorical perceivers performed $67 \%$ correct. At step 8, performance was $87 \%, 73 \%$ and $67 \%$, respectively. Control children performed significantly better than the dyslexic group at step 5 and step 8 (Mann-Whitney U-test $Z=3.26$, $P<0.01$ and $Z=2.62, P<0.01$, respectively). Thus, it appears unlikely that the stimuli were too easy to discriminate for the dyslexic children. 


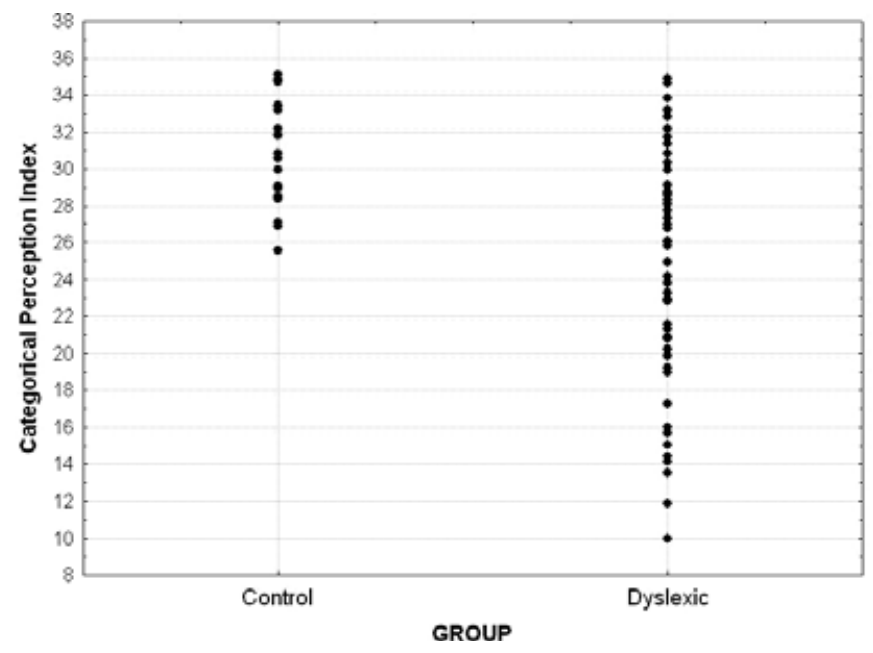

FIG. 5 Categorical perception performance. See Table 1 for computation of the categorical perception index.

\section{Correlations}

No significant correlations were found between MMF values and scores in the behavioural tests.

\section{Discussion}

Dyslexic children and control children did not differ statistically in MMF amplitude. This finding was surprising, as other authors have reported a significantly reduced MMN for dyslexic samples and children with learning disabilities (Kraus et al., 1996; Schulte-Körne et al., 1998, 1999; Baldeweg et al., 1999; Kujala et al., 2001). Because dyslexics are reported to be deficient in categorizing speechsounds (Mody et al., 1997; Adlard \& Hazan, 1998; Serniclaes et al., 2001) and distinguishing CV syllables (Manis et al., 1997), and because the dyslexic children in our sample had significantly lower scores in categorical perception than the control children, we expected to find smaller MMF amplitudes for the dyslexic children compared with controls using the stimulation described. The results obtained here suggest that not all children who have pronounced problems with reading and spelling, as well as categorical perception, have a reduced sensitivity to sound changes that is reflected by an attenuated MMF. Even when the control children were compared with only those dyslexic children who were poor categorical performers, no group differences in MMF amplitude were found. The lack of any correlations between behavioural test performance (including pseudoword reading and the Mottier test, which both reflect phonological abilities) and MMF amplitude also speaks against a relationship between phonological abilities and sensitivity to sound changes. This pattern of results suggests that phonological abilities as well as the competence to identify and categorize speech-sounds can be independent from auditory perception sensitivity. Given the fact that the present sample of dyslexic children $(n=58)$ was considerably larger than most of the samples in other studies examining MMN, it is unlikely that our finding is the result of an insufficient signal-to-noise ratio. We used a standard oddball paradigm with no unusual ISI or stimuli. It therefore seems unlikely that our paradigm was not effective in eliciting a MMF. However, there are very little data published on the magnetic counterpart of the MMN - the MMF - in children. In fact, the only study we found that is comparable in terms of age of the participants was published by Cardy and colleagues (Cardy et al., 2005). However, MMF sizes were not reported.

\section{Stimulus difficulty}

One possible explanation for the absence of MMF differences between the groups might be that the syllables $/ \mathrm{ba} /$ and $/ \mathrm{da} /$ used in the oddball paradigm were too easy to distinguish. The acoustic difference between formants F2 and F3 was $\sim 200 \mathrm{~Hz}$. Kraus and colleagues (Kraus et al., 1996) used an acoustic difference of $80 \mathrm{~Hz}$, and did find MMN differences between good and poor perceivers. However, looking at the categorical perception performance at steps 5 and 8, i.e. the stimuli that were used in the MEG experiment, performance of control children was significantly better than performance of dyslexic children. Therefore, it appears unlikely that the stimuli were too easy to discriminate for the dyslexic children.

Few details were reported of the acoustical parameters of syllables used as stimuli in other studies. No parameters were given for the MMN studies by Schulte-Körne with children (Schulte-Körne et al., 1998) and adults (Schulte-Körne et al., 2001). Thus, the possibility that our contradictory findings are a result of different stimulus parameters cannot be fully ruled out.

Results of other experiments were based on tone stimuli rather than on syllables, e.g. two different tone-patterns (Schulte-Körne et al., 1999), tone-pair reversals (Kujala et al., 2001), tone-patterns with a following tone (Kujala et al., 2003) or sine-tones (Baldeweg et al., 1999), and are thus difficult to compare with the present findings.

\section{Task demand}

Lachmann and colleagues (Lachmann et al., 2005) analysed the MMN in 16 dyslexic and 12 control children. Based on their reading speed in a non-word and a real-word reading test, dyslexic children were separated into subgroups. Children who were significantly slower than controls in only the non-word reading test and who were thus considered to mainly have phonological deficits formed the Dyslexics1 group. Children who were significantly slower than controls in both reading tasks and who were thus considered to have other or more difficulties than only phonological ones formed the Dyslexic-2 group. Syllables $/ \mathrm{ba} /$ and $/ \mathrm{da} /$ were presented in an oddball paradigm, as well as a pair of pure tones with frequencies of 700 and $770 \mathrm{~Hz}$. An attenuated MMN was only found for Dyslexics- 2 in both the tone and the syllable condition. No differences were found between control children and Dyslexics-1. The authors concluded from this finding that children with only phonological problems (Dyslexics-1) do not have problems in basic sound discrimination. The authors also point out that this seemingly contradictory finding might be a consequence of different tasks making different cognitive demands. While graphemephoneme conversion needs to be applied in non-word reading, this is not necessary during an oddball experiment.

Compatible with this argument, one possible explanation of why dyslexic children in the present study did not have attenuated MMF amplitudes might be that they did detect sound changes in the sense that the stimuli were recognized as being 'different' (control-like MMF), but that in an ensuing processing stage the nature of the 'difference' could not be clearly categorized (poor performance in categorical perception). Ramus (2001) states that 'Speech-perception tasks (e.g. [ba]-[da] discrimination) focus most specifically on the sublexical phonological level. Indeed, they only require auditory processing [and] representation at the phonological level [...]. It is not entirely clear whether such tasks propose problems for all dyslexics or just to a subgroup (e.g. Mody et al., 1997).' If this was the case, one might assume that the majority of people with dyslexia do not have a general auditory perceptual deficit. For those who do not it seems more likely that decoding difficulties occur at a later processing step 
when phonemes have to be categorized. Linking our results to the model of lexical access proposed by Ramus (2001) could mean that the acoustic representations after speech input as well as sublexical representations are intact, and that processing difficulties start when access to the phonological lexicon is attempted.

Different task demands might also be an explanation for the contradictory findings between our study and the experiment of Kraus et al. (1996). Our design differed from the one of Kraus et al. in two important points. First, they preselected the children that were examined based on their performance in $/ \mathrm{da} /-/ \mathrm{ga} /$ discrimination. We investigated children that showed marked disturbance in categorical perception involving the identification of the syllables. I.e. the children in the Kraus et al. study had problems discriminating the stimuli, while the children in our study had difficulties identifying the stimuli.

\section{Sample selection}

Schulte-Körne and colleagues (Schulte-Körne et al., 1998) examined a sample of children that was quite similar to ours apart from the age (children were 2-3 years younger in the present study). Only purely dyslexic children with no other impairments were tested. Sample selection is thus an unlikely cause for the different findings between our and Schulte-Körne's study. It is noteworthy, however, that their sample only included 19 dyslexic children, while ours included 53 dyslexic children.

The sample of Kraus et al. (1996) involved children with several types of learning disabilities, including children with ADHD. Our sample only involved dyslexic children with no other significant impairment. It is possible that the children in the present study were relatively less affected than the children in Kraus et al.'s experiment. Nevertheless, one should keep in mind that the MMN findings in Kraus et al.'s study were obtained from a preselected subset of the children who were either good or poor categorical discriminators. Children in the study by Banai and colleagues (Banai et al., 2005) were not preselected in any way. They were also similar to the children in the present study in terms of age. However, as in the study by Kraus et al., the sample consisted of children with learning disabilities rather than dyslexia only. Despite the fact that there was a significant difference in $\mathrm{MMN}$ size and duration on the group level, it is worth pointing out that $72 \%$ of the children had normal MMNs as defined by the authors.

Sample selection might yet be another possibility to explain the lack of MMF attenuation in the present experimental group. Dyslexia is a specific disability of reading and spelling despite appropriate schooling and normal intelligence, without any other sensory or neurological deficits. These diagnostic criteria do not involve any aetiological premises. Therefore, a variety of children might be diagnosed to be dyslexic, without having the same underlying deficit. Dyslexia has been viewed as a heterogeneous disorder that varies to a great extent in strength and expression (Frith, 2001; Demonet et al., 2004). Thus, it is likely that some but not all children have difficulties with basic auditory processing in an unselective sample of dyslexics. But there will also be children whose phonological awareness is reduced without parallel deficient auditory temporal processing (see below). Due to the heterogeneity of the disorder, small samples are at risk for selection bias, which might explain contradictions in results between experiments. Because the sample of the present study was relatively large compared with any other study, it is likely that it is more heterogeneous, but at the same time more representative, than other samples.

Bradlow and colleagues (Bradlow et al., 1999) measured the MMN in a heterogeneous sample of 32 children with learning problems (including learning disability, dyslexia and ADHD) and 72 control children without any preselection. They presented syllables /da/ and /ga/ in an oddball paradigm. The difference in F3 onset frequency between the two syllables was $80 \mathrm{~Hz}$, i.e. syllables were very close to a behavioural discrimination threshold that had been determined beforehand. Two standard/deviant sets were presented, one with a formant transition duration of $40 \mathrm{~Hz}$ and one of $80 \mathrm{~Hz}$. No statistically significant differences in MMN duration and mean amplitude were found for either stimulus set. When only those children were taken into account who either showed very large or very small MMN mean amplitudes, it was revealed that within the group of children with learning problems a greater percentage of children had very small MMN areas than large MMN areas (22 vs. $9 \%$ ) in the $40 \mathrm{~Hz}$ condition, while the reverse was found in the $80 \mathrm{~Hz}$ condition (11 vs. 28\%). Within the control group more children had very large MMN areas than very small MMN areas in both conditions (large MMN: 29 and 28\%; small MMN: 6 and 12\% in the daga $80 \mathrm{~Hz}$ and daga $40 \mathrm{~Hz}$ conditions, respectively). Nevertheless, the majority of children in both groups (61-69\%) showed intermediate MMN areas. Further, there was a high degree of overlap between the groups.

Ramus and colleagues (Ramus et al., 2003) investigated 16 adult dyslexics with an extensive test-battery focusing on phonological, auditory, visual and cerebellar deficits. Besides the fact that the profile of performance and individual deficits varied to a great extent across the subjects, they found that all of the dyslexics had problems with phonology, while only 10 of 16 had deficits with auditory processing. They even consider this as a high incidence compared with other studies that typically find one-third of dyslexics affected on auditory processing (Ramus et al., 2003). It is also stressed that the auditory problems found cannot be characterized as being the consequence of a rapid auditory processing deficit, as the dyslexic subjects did not perform worse on tasks involving rapid auditory processing compared with other auditory tasks. Another important conclusion was that deficient audition leads to poor phonology, but not vice versa. In the light of Ramus et al.'s results, our finding of a 'dissociation' between poor performance in categorical perception (phonology) and controllike MMF amplitudes (audition) seems plausible. The children's auditory systems register the difference between syllables, yet the children cannot categorize them.

\section{Acknowledgements}

This project has been supported by a grant of the Deutsche Forschungsgemeinschaft. We would like to thank all participating children and their parents as well as Professor Quentin Summerfield for helpful comments and proof-reading of the manuscript.

\section{Abbreviations}

ADHD, attention deficit hyperactivity disorder; CV, consonant-vowel; ECG, electrocardiogram; EEG, electroencephalography; EOG, electrooculogram; ISI, interstimulus interval; MEG, magnetoencephalography; MMF, mismatch field; MMN, mismatch negativity; RMS, root mean square.

\section{References}

Adlard, A. \& Hazan, V. (1998) Speech perception in children with specific reading difficulties (dyslexia). Q. J. Exp. Psychol. A, Human Exp. Psychol., 51, 153-177.

Baldeweg, T., Richardson, A., Watkins, S., Foale, C. \& Gruzelier, J. (1999) Impaired auditory frequency discrimination in dyslexia detected with mismatch evoked potentials. Ann. Neurol., 45, 495-503.

Banai, K., Nicol, T., Zecker, S.G. \& Kraus, N. (2005) Brainstem timing: implications for cortical processing and literacy. J. Neurosci., 25, 9850-9857. 
Bradlow, A.R., Kraus, N., Nicol, T.G., McGee, T.J., Cunningham, J., Zecker, S.G. \& Carrell, T.D. (1999) Effects of lengthened formant transition duration on discrimination and neural representation of synthetic CV syllables by normal and learning-disabled children. J. Acoust. Soc. Am., 106, 2086-2096.

Cardy, J.E.O., Flagg, E.J., Roberts, W. \& Roberts, T.P.L. (2005) Delayed mismatch field for speech and non-speech sounds in children with autism. Neuroreport, 16, 521-525.

Demonet, J.F., Taylor, M.J. \& Chaix, Y. (2004) Developmental dyslexia. Lancet, 363, 1451-1460.

Elbro, C., Rasmussen, I. \& Spelling, B. (1996) Teaching reading to disabled readers with language disorders: a controlled evaluation of synthetic speech feedback. Scand. J. Psychol., 37, 140-155.

Farmer, M. \& Klein, R. (1995) The evidence for a temporal processing deficit linked to dyslexia: a review. Psychonomic Bull. Rev., 2, 460-493.

Findeisen, U. \& Melenk, G. (1991) Lauttreue Diktate für die 1. Bis 5. Klasse. Winkler, Bochum.

Frith, U. (2001) What framework should we use for understanding developmental disorders? Dev. Neuropsychol., 20, 555-563.

Grissemann, H. (2000) Zürcher Lesetest (ZLT). Hans Huber, Bern.

Grund, M., Haug, G. \& Naumann, C. (1994) Diagnostischer Rechtschreibtest für 4. Klassen (DRT4). Beltz, Weinheim, Basel.

Heller, K., Kratzmeier, H. \& Lengfelder, A. (1998) Raven-Matrizen-Test. Standard Progressive Matrices (SPM). Deutsche Version. Beltz, Göttingen.

Kraus, N., McGee, T.J., Carrell, T.D., Zecker, S.G., Nicol, T.G. \& Koch, D.B. (1996) Auditory neurophysiologic responses and discrimination deficits in children with learning problems. Science, 273, 971-973.

Kujala, T., Belitz, S., Tervaniemi, M. \& Naatanen, R. (2003) Auditory sensory memory disorder in dyslexic adults as indexed by the mismatch negativity. Eur. J. Neurosci., 17, 1323-1327.

Kujala, T., Karma, K., Ceponiene, R., Belitz, S., Turkkila, P., Tervaniemi, M. \& Näätänen, R. (2001) Plastic neural changes and reading improvement caused by audiovisual training in reading-impaired children. Proc. Natl Acad. Sci. USA, 98, 10509-10514.

Lachmann, T., Berti, S., Kujala, T. \& Schröger, E. (2005) Diagnostic subgroups of developmental dyslexia have different deficits in neural processing of tones and phonemes. Int. J. Psychophysiol., 56, 105-120.

Manis, F.R., McBride-Chang, C., Seidenberg, M.S., Keating, P., Doi, L.M., Munson, B. \& Petersen, A. (1997) Are speech perception deficits associated with developmental dyslexia? J. Exp. Child. Psychol., 66, 211-235.

McAnally, K.I., Hansen, P.C., Cornelissen, P.L. \& Stein, J.F. (1997) Effect of time and frequency manipulation on syllable perception in developmental dyslexics. J. Speech, Language, Hearing Res., 40, 912-924.

Mody, M., Studdert-Kennedy, M. \& Brady, S. (1997) Speech perception deficits in poor readers: auditory processing or phonological coding? J. Exp. Child. Psychol., 64, 199-231.

Müller, R. (1997) Diagnostischer Rechtschreibtest für 3. Klassen (DRT3). Beltz, Weinheim, Basel.
Rack, J.P., Snowling, M.J. \& Olson, R.K. (1992) The nonword reading deficit in developmental dyslexia: a review. Reading Research Q., 27, $29-53$.

Ramus, F. (2001) Outstanding questions about phonological processing in dyslexia. Dyslexia, 7, 197-216.

Ramus, F., Rosen, S., Dakin, S.C., Day, B.L., Castellote, J.M., White, S. \& Frith, U. (2003) Theories of developmental dyslexia: insights from a multiple case study of dyslexic adults. Brain, 126, 841-865.

Schulte-Körne, G., Deimel, W., Bartling, J. \& Remschmidt, H. (1998) Auditory processing and dyslexia: evidence for a specific speech processing deficit. Neuroreport, 9, 337-340.

Schulte-Körne, G., Deimel, W., Bartling, J. \& Remschmidt, H. (1999) Preattentive processing of auditory patterns in dyslexic human subjects. Neurosci. Lett., 276, 41-44.

Schulte-Körne, G., Deimel, W., Bartling, J. \& Remschmidt, H. (2001) Speech perception deficit in dyslexic adults as measured by mismatch negativity (MMN). Int. J. Psychophysiol., 40, 77-87.

Serniclaes, W., Sprenger-Charolles, L., Carre, R. \& Demonet, J.F. (2001) Perceptual discrimination of speech sounds in developmental dyslexia. J. Speech, Language, Hearing Res., 44, 384-399.

Shaywitz, S.E. (1996) Dyslexia. Scientific Am., 275, 98-104.

Stanovich, K.E. (1988) The right and wrong places to look for the cognitive locus of reading disability. Ann. Dyslexia, 38, 154-177.

Swan, D. \& Goswami, U. (1997) Phonological awareness deficits in developmental dyslexia and the phonological representations hypothesis. J. Exp. Child. Psychol., 66, 18-41.

Tallal, P. (1980) Auditory temporal perception, phonics, and reading disabilities in children. Brain Language, 9, 182-198.

Tallal, P., Miller, S.L., Bedi, G., Byma, G., Wang, X., Nagarajan, S.S., Schreiner, C., Jenkins, W.M. \& Merzenich, M.M. (1996) Language comprehension in language-learning impaired children improved with acoustically modified speech. Science, 271, 81-84.

Temple, E., Poldrack, R.A., Salidis, J., Deutsch, G.K., Tallal, P., Merzenich, M.M. \& Gabrieli, J.D. (2001) Disrupted neural responses to phonological and orthographic processing in dyslexic children: an fMRI study. Neuroreport, 12, 299-307.

Torgesen, J.K., Wagner, R.K. \& Rashotte, C.A. (1994) Longitudinal studies of phonological processing and reading. J. Learning Disabilities, 27, 276-286; discussion 287-291.

Vellutino, F.R. (1987) Dyslexia. Scientific Am., 256, 34-41.

Welte, V. (1981) Der Mottiertest, ein Prüfungsmittel für die Lautdifferenzierungsfähigkeit und die auditive Merkfähigkeit. Sprache-StimmeGehör, 5, 121-125.

Wright, B.A., Lombardino, L.J., King, W.M., Puranik, C.S., Leonard, C.M. \& Merzenich, M.M. (1997) Deficits in auditory temporal and spectral resolution in language-impaired children. Nature, 387, $176-178$. 